\title{
Liquid Crystal Droplet Patterns to Monitor Catalase Activity at Femtomolar Levels
}

\author{
Stephanie Yoon and Chang-Hyun Jang* \\ Department of Chemistry, Gachon University, Seongnam 461-701, Korea. E-mail: chjang4u@gachon.ac.kr \\ Received April 11, 2014, Accepted May 14, 2014
}

\begin{abstract}
Catalase (CAT) decomposes hydrogen peroxide that is toxic to the body. In this study, simple and sensitive detector has been developed for observing catalase activity using liquid crystal droplet system. Microscale LC droplet patterns are formed by spreading aldehyde-doped nematic liquid crystal on pre-treated glass slides. When hydrogen peroxide is added, aldehyde is oxidized and amphiphiles are formed. Dodecanoates cause the pattern to transit from bright to dark as they self-assemble to form a carboxyalte monolayer at the interface. When a drop of pre-incubated CAT and hydrogen peroxide mixture is placed onto the pattern, bright fan-shape is observed. This planar optical appearance indicates that catalase has decomposed hydrogen peroxide. Compared to the detectors that have been previously developed, this system is more sensitive with detection limit of $1 \mathrm{fM}$. This research suggests further studies to be on LC droplet patterning to develop highly sensitive and methodologically simple sensors for various chemicals.
\end{abstract}

Key Words : Liquid crystal, 4-Cyano-4'-pentylbiphenyl (5CB), Droplet pattern, Catalase, Hydrogen peroxide

\section{Introduction}

All aerobic organisms use cellular respiration to convert ingested nutrients into usable energy in the body; in which process, hydrogen peroxide is a harmful by-product. ${ }^{1}$ Hydrogen peroxide, a toxic chemical, can lead to cellular damage, especially to the mitochondria. Though its toxicity, hydrogen peroxide contributes to many pathways in mammalian cells, such as defense mechanism against pathogens ${ }^{2}$ and as a signaling molecule to regulate cellular growth, differentiation, migration, and apoptosis. ${ }^{3}$ However, little is known about the mechanisms of how hydrogen peroxide work as a important signaling molecule. ${ }^{4}$ Understanding cellular signaling process is crucial to understand organisms, and therefore, detection of hydrogen peroxide calls for a great attention. Detection techniques for hydrogen peroxide has been developed throughout the years, including titrimetry, spectrometry, chemiluminescence, and electrochemistry. A hydrogen peroxide sensor using electrochemistry has overcame many drawbacks of previous methods. ${ }^{5}$ Fast determination of hydrogen peroxide is beneficial in many areas ranging from medicine to food control to development of various biosensors; thus leading many scientists' attention to the study of electrochemical hydrogen peroxide detector. ${ }^{6,7}$ One of the developed hydrogen peroxide sensors is using direct electrochemistry of catalase at amine-functionalized graphene/gold nanoparticles composite film. ${ }^{8}$ And yet another sensor determined amount of hydrogen peroxide from the amount of oxygen released when catalase-collagen membrane is placed in $\mathrm{H}_{2} \mathrm{O}_{2}$ solution. ${ }^{9}$

Catalase is a tetrameric enzyme that occurs in almost all aerobic organisms to decompose hydrogen peroxide into water and oxygen to protect the cells from oxidative stress. ${ }^{10}$ Catalase is one of the main enzymes that regulate the accumulation of hydrogen peroxide, meaning catalase detection alone can be useful in finding signaling pathway of hydrogen peroxide. Not only for understanding how hydrogen peroxide works as intracellular signaling molecule, but catalase itself also has much importance clinically. Patients lacking catalase usually exhibit only mild symptoms and these patients are called to have a disease called acatalasia, which is thought to be benign. ${ }^{11}$ However, some research has suggested possible outcomes of catalase deficiency. In a series of Hungarian patients with catalase deficiency, there was a higher frequency of diabetes, suggesting an association between diabetes and acatalasia. ${ }^{12}$ Another study published a paper mentioning catalase deficiency as a possible cause to make kidneys more at risk to oxidant tissue injury and renal fibrosis in mice. ${ }^{13}$

For one reason or the other, detection of catalase has been studied in recent years. An aptamer based SPR biosensor has been developed for the detection of catalase in milk samples since catalase is an indicator of mastitis disease in milk. ${ }^{14} \mathrm{By}$ measuring the degradation of hydrogen peroxide indirectly, Futo et al. developed an amperometric based biosensor for catalase detection in milk samples. ${ }^{15}$ In another study, photoelectrochemical sensor for catalase activity was developed with a detection limit of $76 \mathrm{pM}^{16}$ Taking out all complex and time-consuming methods, liquid crystal sensor to observe catalase activity at aqueous/LC interface was also developed. ${ }^{17}$

Nematic liquid crystals is a material that is mostly rod-like that has some characteristics of liquid, being long-range orientational order, and another characteristic of solid, being short-range positional order. ${ }^{18}$ Such material has an ability to amplify biomolecular events and to convert them into optical signal visible by the naked eye under a microscope, ${ }^{19}$ which is a great innovation for the scientists of the field. The orientation of liquid crystals quickly responds to a stimulus ${ }^{20}$ and this orientational change holds a great promise for sensing application. ${ }^{21}$

Using this promising detection material, liquid crystal sensor for catalase enzyme has already been developed in our lab. 
Though aqueous/LC interface system provided sensitive detection scheme of catalase with $1 \mathrm{nM}$, it involved drying time, grids and optical cells with chambers that complicated and elongated the procedure. ${ }^{17}$ To simplify the procedure and increase the sensitivity of the detection by increasing surface area, we incorporated previous report on micrometerscale LC droplets with distinct optical texture that can be used as detection scheme..$^{22}$ The LC droplets were produced by dissolving LCs in organic solvent and then dropping the solution onto a glass slide. As the organic solvent evaporated and the LC droplets spread uniformly on the surface, distinct pattern showed and was ready for experimental use.

Using this much advantageous detection mechanism of LC droplet system, we developed a quick and simple catalase detector. Dark-cross pattern (Fig. 1(b)) was observed when hydrogen peroxide was applied onto the pattern, representing perpendicular orientation. When hydrolyzed-hydrogen peroxide was made in contact with the pattern, it displayed bright fan shape (planar orientation) (Fig. 1(a)). This indicated that enzymatic reaction between catalase and its substrate, hydrogen peroxide, was successfully carried, decomposing hydrogen peroxide to a significant level to block the orientational change inducement.

\section{Experimental}

Materials. Nematic liquid crystal (LC) 4-cyano-4'-pentylbiphenyl (5CB) manufactured by BDH Chemicals from EM industries (Hawthorne, NY) was used in the experiment. Premium glass microscope slides were purchased from Fisher Scientific (Pittsburg, PA) and used. $n$-heptane (anhydrous) from Daejung Chemicals \& Metals Co. Ltd. (South Korea) was used to dissolve $5 \mathrm{CB}$. Sulfuric acid, hydrogen peroxide $(30 \% \mathrm{w} / \mathrm{v})$, octyltrichlorosilane (OTS), and phosphate buffered saline (PBS), dodecanal, hydrogen peroxide, and catalase were all from Sigma-Aldrich. Otherwise mentioned, all aqueous solution were prepared with deionized water (18 M $\Omega$ $\mathrm{cm}$ ) using a Milli-Q water purification system (Millipore, Bedford, MA).

Treatment of Glass Microscope Slides (with Piranha Solution, then with OTS). Glass microscope slides were immersed in piranha solution composed of $30 \% \mathrm{H}_{2} \mathrm{O}_{2}$ and $70 \% \mathrm{H}_{2} \mathrm{SO}_{4}$, for $30 \mathrm{~min}$ at $80{ }^{\circ} \mathrm{C}$ (CAUTION: since piranha solution reacts violently with organic compounds, you need extreme caution in handling it. DO NOT store the solution in a closed container). After heating, they were cooled at room temperature for half an hour. The slides were then rinsed with DI water, ethanol, and methanol, 3 times per solvent type and in the given order. Cleaned piranha glasses were dried under $\mathrm{N}_{2}$ gas, then heated/stored at $120^{\circ} \mathrm{C}$ oven for overnight. After heating, they were immersed in OTS $/ n$ heptane solution for $30 \mathrm{~min}$. Following OTS deposition, the slides were rinsed 3 times using dichloromethane and dried with $\mathrm{N}_{2}$ gas, now ready for experimental use. Prepared OTS slides should be stored at room temperature.

Preparation of Large Scale LC Droplet Pattern. Approximately $2.1 \mathrm{wt} \%$ dodecanal-doped $5 \mathrm{CB}$ was prepared and with this, $1 \%(\mathrm{v} / \mathrm{v})$ aldehyde-doped $5 \mathrm{CB}$ to heptane solution was made. $2 \mu \mathrm{L}$ of this solution was dropped onto OTStreated glass slide to make a micro-scale pattern. As the organic solvent evaporates, the pattern stabilizes and ready for further experiments to proceed. $3 \mu \mathrm{L}$ of aqueous solution of interest was introduced to the LC droplet pattern at room temperature for observation. Freshly doped 5CB and freshly made $5 \mathrm{CB}+$ heptane solutions are required for stable pattern formation. The temperature also plays a role in pattern stability, therefore, it is important to keep the temperature to room temperature of $25^{\circ} \mathrm{C}$. The pattern is made by human hands and mechanical system of producing uniformly sized droplet has not yet been developed. However, skilled hands can form and reproduce similar-sized, well-distributed droplet pattern. Because we only see a small portion of the pattern, it is easy to find uniformly patterned-spot and restrict the experiment to be on that spot.

Preparation of CAT Solution. Enzyme catalase has solubility of $1 \mathrm{mg} / \mathrm{mL}$ in $50 \mathrm{mM}$ PBS. High concentration of PBS can possibly affect the orientation of LC droplet pattern due to salt ion interaction at the interface. However, CAT is only dissolved at $50 \mathrm{mM}$ PBS, relatively concentrated PBS. Therefore, it first needs to be completely dissolved in 50 mM PBS, and then it is diluted using DI water to bring both PBS concentration and CAT concentration down to the desired concentrations.

Preparation of a Mixture of CAT and Hydrogen Peroxide. Double the CAT concentration of desired concentration in the mixture was prepared separately. $0.2 \%$ hydrogen peroxide was prepared separately. Then $500 \mu \mathrm{L}$ of prepared CAT solution and $500 \mu \mathrm{L}$ of prepared hydrogen peroxide were mixed together to form $1 \mathrm{~mL}$ of a mixture of desired concentration CAT and $0.1 \% \mathrm{H}_{2} \mathrm{O}_{2}$. When the concentration of CAT was lowered much, the solution was incubated in a $40{ }^{\circ} \mathrm{C}$-oven for $2 \mathrm{~h}$. This was done to help the enzymatic reaction by fixing the reaction temperature to be at enzyme's optimal temperature of $40{ }^{\circ} \mathrm{C}$.

Optical Examination of LC. The analyzer in our polarized light microscope (ECLIPESE LV100POL, Nikon, Tokyo, Japan) was fixed to be 90 degrees to the polarizer of the microscope. Then, using the microscope, we transmitted the polarized light through the LC droplet pattern. Optical textures of $5 \mathrm{CB}$ were imaged using a digital camera (DS$2 \mathrm{Mv}$, Nikon, Tokyo, Japan) attached to the microscope. We used $4 \times$ objective lens between crossed polarizers to observe the image. To correctly compare and contrast images, all images had a resolution of $1600 \times 1200$ pixels, a gain of $1.00 \times$, and a shutter speed of $1 / 10 \mathrm{~s}$.

\section{Results and Discussion}

Optical Response of the Aldehyde-Doped 5CB to Hydrogen Peroxide Shown as Patterns. Scholars are bringing close attentions to recent studies of simple and sensitive detection scheme utilized by liquid crystal droplet pattern system. This innovative system uses various methods to change liquid crystal orientation and develop them into 
biosensors. When we use doped LC with functional materials instead of pure LC, this system, even more so, broadens the range of sensing applications. ${ }^{23,24}$ Detection that could not be formulated using pure 5CB was enabled using PBA-doped $5 \mathrm{CB}$ and $\mathrm{pH}$ sensor was successfully built. ${ }^{23}$ In another study, enzymatic activity of lipase was studied using glyceryl trioleate doped in $5 \mathrm{CB} .{ }^{25}$ Understanding feasibility of doping technique, we used aldehyde-doped $5 \mathrm{CB}$ as detection material in our research. If randomly arranged aldehyde can be oriented in one direction at the surface level, the long hydrocarbon chain attached will also induce $5 \mathrm{CB}$ to change the orientation with it. This formulates a change in the optical image of liquid crystal droplet pattern and allows observation.

Increasing $\mathrm{pH}$ from 4.0 to 5.0 , dodecanoic acid doped in $5 \mathrm{CB}$ underwent an orientational change in a previous study. ${ }^{23}$ As the $\mathrm{pH}$ was raised, dodecanoic acid was deprotonated and formed dodecanoate, which is an amphiphile. Increased concentration of dodecanoates in LC successfully allowed self-assembly of amphiphiles at aqueous/LC interface, with hydrophilic head group facing the aqueous side and hydrophobic chains of carboxylates facing the LC. Interaction between dodecanoate's long chain and 5CB has induced a change in optical image of LCs from bright to dark, indicating an orientational change from planar to homeotropic. Combining this result and another research result of 3\% hydrogen peroxide ( $\mathrm{pH} 11$ ) oxidizing aldehydes into carboxylic acid, ${ }^{26}$ we formulated a hypothesis that an orientational transition would happen when hydrogen peroxide is added to dodecanaldoped 5CB. This is also the same background information another research followed to make a LC interfacial biosensor for hydrogen peroxide. Oxidation reaction will cause dodecanal to become dodecanoic acid, and formed dodecanoic acid will be deprotonated spontaneously, allowing LC orientation shift from planar (Fig. 1(a)) to homeotropic (Fig. 1(b)) at the interface.

LC droplet pattern was formed by dropping $1 \%(\mathrm{v} / \mathrm{v})$ aldehyde-doped 5CB and heptane solution onto OTS glass slide. The solution spread on the slide and then organic solvent evaporated, forming pattern with dark cross image under polarized microscope. This image is consistent with the one from a previous study showing dark cross appearance when pure 5CB pattern was in contact with the air. ${ }^{22}$ We then experimented how hydrogen peroxide will change the pattern as it comes in contact with the pattern. At first, we selected $3 \%$ hydrogen peroxide to check if oxidation of aldehydes carries out successfully. When we introduced $3 \%$ hydrogen peroxide to the dodecanal-doped $5 \mathrm{CB}$, bright fan-like shaped patterns appeared immediately, indicating planar orientation of liquid crystals (Fig. 2(a)). The image shows some patterns that are perfectly fan-shaped while some are distorted fan shaped. This is for yet unknown reason but they are assumed to be both planar orientation due to similar shape and brightness. When $5 \mathrm{~min}$ have passed, hydrogen peroxide has successfully induced an orientational change of LCs. Liquid crystals changed their orientations from planar to perpendicular, causing the optical image to be now, dark cross-shaped (a)

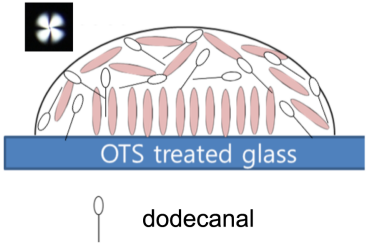

Figure 1. Schematic illustration of orientational change of LCs as applied hydrogen peroxide oxidizes dodecanal to its carboxylate form: (a) planar orientation and (b) perpendicular orientation.

ones (Fig. 2(b)). Interestingly, in this image shown, the pattern shapes do not show up clearly and distinctively as the patterns in contact with the air alone. However, we can conclude that the orientational change took place since there was a drastic change in the images in their brightness. When the pattern was viewed under a higher optical power, the dark cross patterns were more distinctively shown. The image shows only as dark due to certain power and certain brightness chosen for the experiment, but the transition was evidently induced.

Hydrogen peroxide experiment was a required step prior to the observation of catalase enzymatic reaction. In order to develop highly sensitive catalase detector, it is important to lower hydrogen peroxide concentration as much as possible and then, use that specific concentration for following catalase reaction. Thus, we determined the detection limit of hydrogen peroxide using this system. $0.3 \%$ hydrogen peroxide was applied to the pattern and it appeared bright (Fig. 2(c)). Bright fan-shaped pattern remained, but when 12 min have passed, complete dark cross pattern was observed (Fig. 2(d)), indicating a shift from planar to homeotropic orientation.

To further decrease the detection limit of hydrogen peroxide, we then introduced $0.03 \%$ hydrogen peroxide onto aldehydedoped 5CB pattern. The pattern appeared bright initially (Fig. 2(e)) and it started to undergo a transition to dark but at a much slower rate than happened in higher concentrations. When 15 min passed, the pattern was still only partially dark (Fig. 2(f)), indicating coexistance of both planar and perpendicular orientations. Though $0.03 \%$ hydrogen peroxide slowly but surely oxidized the aldehyde and led to orientational change, for our experimental purpose to further detect catalase activity, we restricted time so that the orientational shift will happen within $12 \mathrm{~min}$. Therefore, though aldehyde-doped 5CB can sense hydrogen peroxide concentration with detection limit of $0.03 \%$, we higher the concentration to $0.1 \%$ hydrogen peroxide and tried whether $0.1 \%$ hydrogen peroxide can induce an orientation change within the time frame.

First, to confirm that adding any aqueous phase alone does not induce an orientational change, we carried out a control experiment with DI water. When DI water was added to the pattern, it appeared bright (Fig. 3(a)) and continued to sustain the bright fan-shaped pattern shown in random orientation for $20 \mathrm{~min}$ (Fig. 3(b)). This control shows that if there is any transition of the patterns, it must be hydrogen peroxide components causing the phenomenon rather than the phase of 


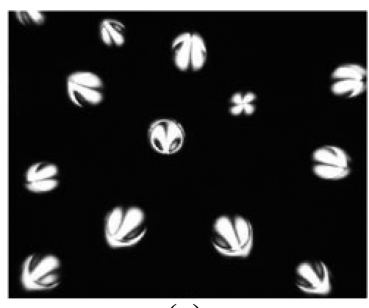

(a)

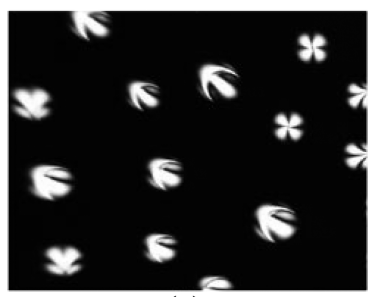

(c)

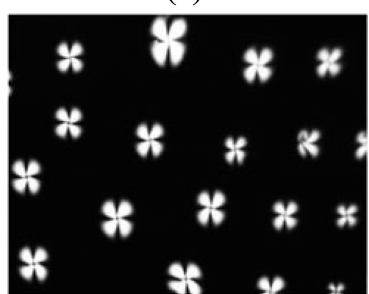

(e)

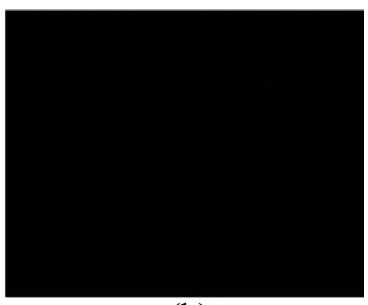

(b)

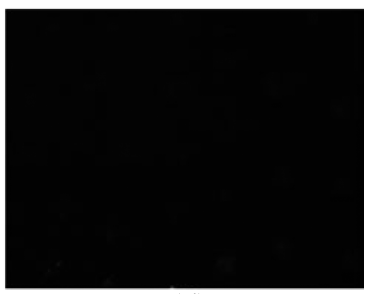

(d)

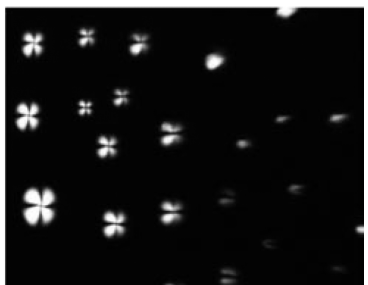

(f)
Figure 2. Polarized light microscopy images of dodecanal-doped $5 \mathrm{CB}$ droplet pattern: in contact with: $3 \%$ hydrogen peroxide was added to aldehyde-doped 5CB droplet pattern for (a) 1 min and (b) $5 \mathrm{~min}, 0.3 \%$ hydrogen peroxide for (c) $1 \mathrm{~min}$ and (d) $12 \mathrm{~min}$, and $0.03 \%$ hydrogen peroxide for (e) $1 \mathrm{~min}$ and (f) $15 \mathrm{~min}$.

added solution. In previous LC sensor, hydrogen peroxide concentration used was $0.1 \%$ with $\mathrm{pH}$ controlled to $11 .{ }^{17}$ Since we have greatly increased surface areas of detection and already seeing the capability of even lower concentration of hydrogen peroxide inducing a change, we tried our experiments with $0.1 \%$ hydrogen peroxide without $\mathrm{pH}$ controlled. When we transferred $0.1 \%$ hydrogen peroxide to aldehydedoped $5 \mathrm{CB}$, bright fan-shaped was observed at first (Fig. $3(\mathrm{c}))$. This pattern means LCs are randomly distributed in the droplet. Within $5 \mathrm{~min}$, the pattern was undergoing a transition and appeared partially dark (Fig. 3(d)). Finally, when the image was captured at $12 \mathrm{~min}$ after the reaction first started, the pattern was completely changed to dark cross-shaped (Fig. 3(e)), representing homeotropic orientation. The distinct change happened without $\mathrm{pH}$ control already proves to us that the sensitivity has been upgraded from that of LC-sensor using the interface. Therefore, $0.1 \%$ hydrogen peroxide was the concentration selected to carry further experiments for catalase detection.

In order to have successful results, we need the aldehydes to be oxidized to carboxylic acid and for the carboxylic acid to be deprotonated to our final desired product of carboxylate, which can orient itself in response to chemical environment. The last step of this chemical reaction, deprotonation, happens much faster in alkaline environment since carboxylic acid more easily gives up its proton to base in the solvent. Also oxidation reaction can be expedited by addition of

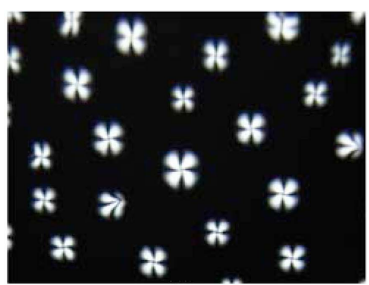

(a)

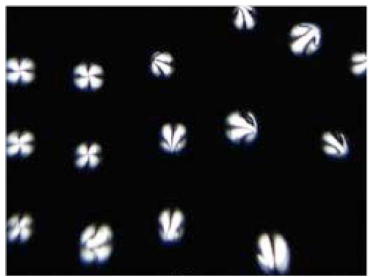

(c)

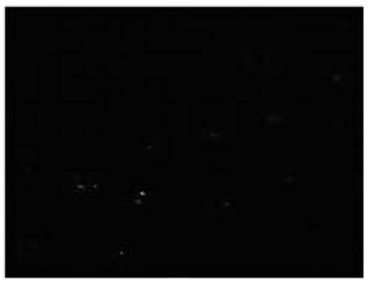

(e)

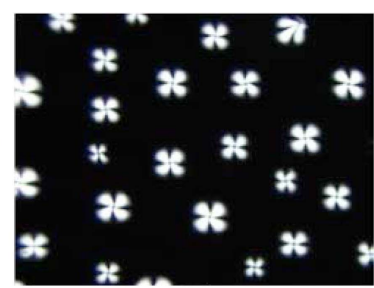

(b)

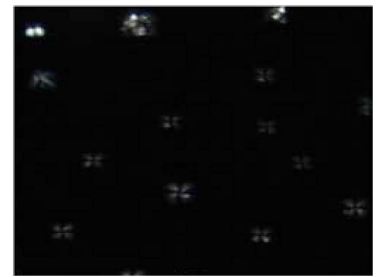

(d)

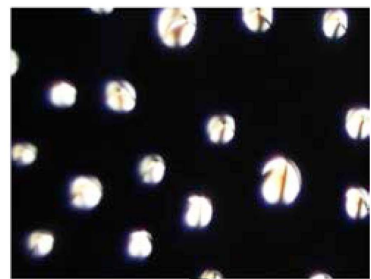

(f)
Figure 3. Polarized light microscopy images of LCs: DI water formed an aqueous phase over dodecanal-doped 5CB droplets, for (a) $1 \mathrm{~min}$ and (b) $20 \mathrm{~min}$. After $0.1 \%$ hydrogen peroxide was added to the dodecanal-doped $5 \mathrm{Cb}$, (c) $1 \mathrm{~min}$, (d) $5 \mathrm{~min}$, and (e) 12 min past. As control, the image shows (f) $20 \mathrm{~min}$ after $0.1 \%$ hydrogen peroxide was added to pure $5 \mathrm{CB}$ pattern.

$\mathrm{NaOH}$ since hydrogen peroxide becomes more nucleophilic in alkaline environment. Using this information, in previous study, LC interface that could not formulate a transition with $0.3 \%$ hydrogen peroxide showed a transition when $\mathrm{pH}$ was controlled to $11 .{ }^{17}$ Thus, if we were to control the $\mathrm{pH}$ of the system as well, the detection limit of hydrogen peroxide could go down even more. Though we did not try alkaline environment due to possible yet unwanted reaction of $\mathrm{NaOH}$ inducing orientational change of LCs, theoretically, alkaline environment should help lowering hydrogen peroxide detection limit.

As a control experiment to test that hydrogen peroxide alone does not change LC orientation but it does so via oxidizing aldehydes in doped $5 \mathrm{CB}$, we carried out $0.1 \%$ hydrogen peroxide application on pure $5 \mathrm{CB}$ droplet pattern. When $0.1 \%$ hydrogen peroxide was added to the pattern and $1 \mathrm{~min}$ has passed, pure $5 \mathrm{CB}$ presented planar orientation through bright fan-shaped pattern. Even after $20 \mathrm{~min}$ have passed, the pattern remained bright fan-shaped (Fig. 3(f)), indicating no transition appeared. The shape of the droplets in this image is slightly crooked from the normal fan shaped patterns but the brightness indicates that it is not homeotropic orientation, which is shown by dark cross shape. This distorted fan shapes are a result of unknown reason but it is assumed to be planar orientation in accordance with past $\mathrm{LC}$ research. This shows that the change made in $0.1 \%$ hydrogen peroxide 
addition to aldehyde-doped 5CB is by oxidation of aldehydes, not by simply adding hydrogen peroxide. Interestingly, pattern formed by $0.1 \%$ hydrogen peroxide on pure $5 \mathrm{CB}$ was bright as in planar orientation but the shape was not perfectly fan shaped. Aldehyde-doped pattern sometimes had a mixture of perfect fan shape and distorted fan shape or all perfect fan shape.

Patterns in Response to the Enzymatic Interaction Between Catalase and Hydrogen Peroxide. If hydrogen peroxide concentration is lowered, the detection limit of catalase will also be lowered. However, because we wanted to prove to the scientists how droplet system alone has increased sensitivity of catalase, we used $0.1 \%$ hydrogen peroxide, the same concentration as the one used in aqueous/LC interface sensor. ${ }^{17}$ With everything else constant, we would be able to show the sole benefit of droplet system and its potential to be developed as biosensor.

Another reason we chose $0.1 \%$ hydrogen peroxide is because we did not want to leave any questionable remarks at our conclusion. If we were to use this system for detecting different enzyme such as urease, positive activity of urease will change the orientation of LCs to be perpendicular. ${ }^{27}$ However, in our experiment, the substrate changes the orientation of LCs instead of the enzyme reacting with the substrate inducing the transition. Therefore, if the planar orientation is remained throughout, we cannot conclude whether it is due to catalase addition alone or because hydrogen peroxide was successfully decomposed by catalase. By using relatively high hydrogen peroxide concentration, we wanted to confirm that catalase activity of hydrolyzing hydrogen peroxide was successfully carried out and that not a significant amount of hydrogen peroxide is left to cause an orientational change.

For these two reasons, $0.1 \%$ hydrogen peroxide concentration was determined. As discussed in the previous section when $0.1 \%$ hydrogen peroxide alone was added to the pattern for $20 \mathrm{~min}$, the pattern was completely dark (Fig. 4(a)), indicating homeotropic orientation of doped LCs. This proves a change in patterns has occurred after a significant amount of time has passed. We pre-incubated an aqueous mixture of $1 \mathrm{nM} \mathrm{CAT}$ and $0.1 \%$ hydrogen peroxide at room temperature for $45 \mathrm{~min}$. This solution was transferred onto the doped LC pattern and observed. At $1 \mathrm{~min}$, bright fan-shaped pattern appeared (Fig. 4(b)). The pattern remained as such at $20 \mathrm{~min}$ mark (Fig. 4(c)), maintaining the planar orientation of LCs. There was no change in pattern until the solution completely evaporated. This was consistent result as to when DI water was introduced to the aldehyde-doped 5CB pattern. Even though $0.1 \%$ hydrogen peroxide was present in the solution at first, which is enough to induce an orientational change of LCs, there was no transition, indicating catalase has decomposed hydrogen peroxide to a significant degree.

Detection Limit of Catalase. After we confirmed the catalase enzymatic activity of decomposing hydrogen peroxide, we proceeded to find the detection limit for the enzyme. $1 \mathrm{nM}$ of catalase successfully decomposed hydrogen peroxide and inhibited hydrogen peroxide from inducing a change in the LC droplet patterns. By increasing the surface area of detection material in contact with the experimental solution from the previous detection scheme of LC/ aqueous interface system, we hypothesized that the detection limit of catalase would be much lowered. For the enzyme catalase to be active at the best conditions, optimal temperature of $40{ }^{\circ} \mathrm{C}$ was selected for the enzymatic reaction. We pre-incubated a solution of $1 \mathrm{pM}$ CAT-hydrolyzed $0.1 \%$ hydrogen peroxide for $1 \mathrm{~h}$ at $40{ }^{\circ} \mathrm{C}$ and then transferred a small amount of it to aldehyde-doped 5CB. Hydrogen peroxide was decomposed and bright fan-shaped pattern was observed at 12 min mark (Fig. 5(a)). This figure does not show the perfect fan shape but the planar orientation is confirmed by the brightness. This discrepancy in shapes of planar orientation has not been identified with a reason. Then we performed the same experiment using a 10-fold diluted concentration of CAT. For $2 \mathrm{~h}, 100 \mathrm{fM}$ CAT and $0.1 \%$ hydrogen peroxide was mixed and pre-incubated at $40{ }^{\circ} \mathrm{C}$. Taking $3 \mu \mathrm{L}$ of this aqueous solution and dropping it onto the pattern, bright fan-shaped appeared at first (Fig. 5(b)). The bright pattern was maintained for 12 min of reaction time (Fig. 5(b)). This means the planar orientation remained from the beginning of the reaction, also referring to the fact that the hydrolysis of hydrogen peroxide was carried out. When we once again lowered catalase concentration by another 10-fold to $10 \mathrm{fM}$, the orientation remained planar from the beginning to the end of 12 min (Fig. 5(c)).

When $1 \mathrm{fM}$ CAT and $0.1 \%$ hydrogen peroxide was preincubated for $2 \mathrm{~h}$ at $40{ }^{\circ} \mathrm{C}$ and added onto the pattern, it carried planar orientation, indicated as bright fan-shaped. When 12 min passed, bright fan-shaped remained partially and some parts started to undergo an orientational transition (Fig. 5(d)). This indicated that there is some hydrogen peroxide left not decomposed, inducing an orientational change

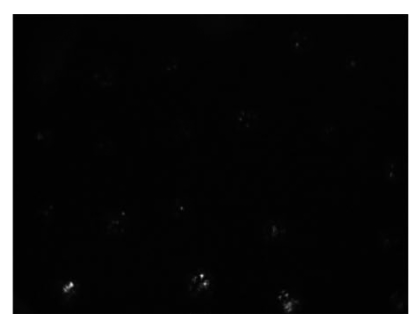

(a)

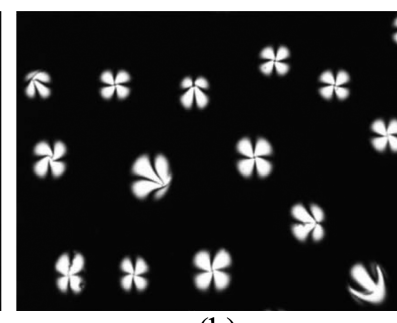

(b)

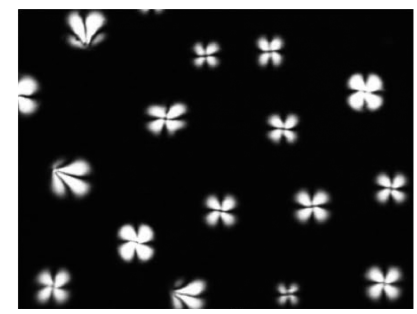

(c)

Figure 4. Optical image of aldehyde-doped 5CB droplet: (a) in contact with $0.1 \%$ hydrogen peroxide for 20 min, in contact with $1 \mathrm{nM}$ CAT-hydrolyzed $0.1 \%$ hydrogen peroxide for (b) $1 \mathrm{~min}$, (c) $20 \mathrm{~min}$ 


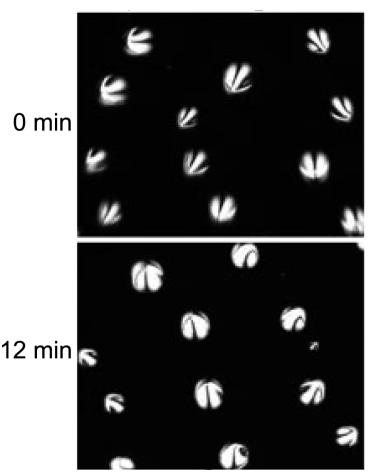

(a)

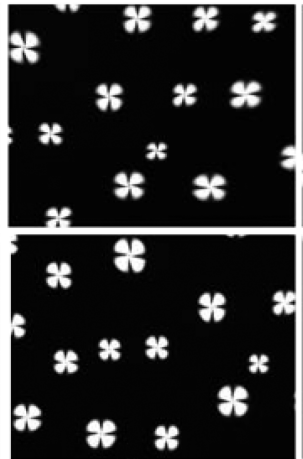

(b)

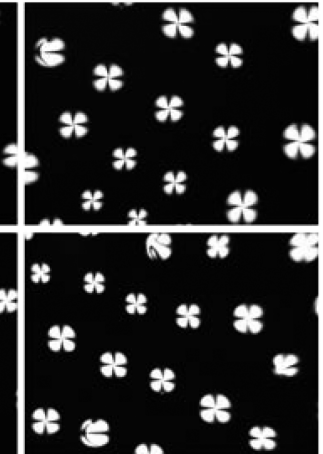

(c)

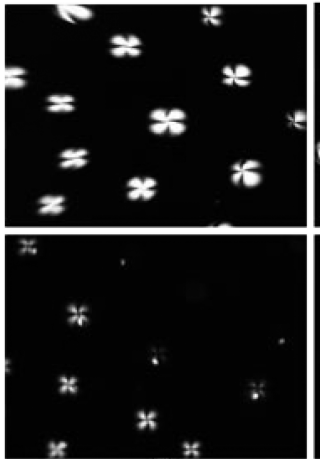

(d)

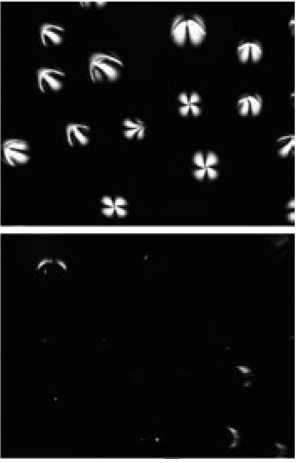

(e)

Figure 5. Patterns produced by aldehyde-doped 5CB droplet as it was in contact with: (a) 1 pM-CAT-hydrolyzed $0.1 \%$ hydrogen peroxide, (b) $100 \mathrm{fM}$-CAT-hydrolyzed $0.1 \%$ hydrogen peroxide, (c) $10 \mathrm{fM}$-CAT-hydrolyzed $0.1 \%$ hydrogen peroxide, (d) $1 \mathrm{fM}-\mathrm{CAT}$-hydrolyzed $0.1 \%$ hydrogen peroxide, and (e) $0.1 \mathrm{fM}$-CAT-hydrolyzed $0.1 \%$ hydrogen peroxide.

from planar to perpendicular. $0.1 \%$ hydrogen peroxide started to change from bright fan shape to dark cross in a few min but in this case, $1 \mathrm{fM}$ CAT-hydrolyzed $0.1 \%$ hydrogen peroxide started to undergo a transition around $10 \mathrm{~min}$ and showing this partiality at 12 min mark. This showed the concentration of hydrogen peroxide left in this solution must be less than $0.1 \%$ and therefore indicating $1 \mathrm{fM}$ CAT was enough to carry some decomposition of hydrogen peroxide. Another 10 -fold dilution was made and tested to confirm the detection limit. When $0.1 \mathrm{fM}$ CAT and $0.1 \%$ hydrogen peroxide was pre-incubated and applied onto the pattern, at first bright fan shaped pattern was observed. 12 min later, it changed completely dark (Fig. 5(e)), indicating hydrogen peroxide was not decomposed at a significant level. This series of experiments concludes the detection limit of this system to be $1 \mathrm{fM}$ and once again confirmed that bright-to-dark shift in patterns is caused solely by hydrogen peroxide at the aqueous interface. The detection limit could be further lowered by using less concentrated hydrogen peroxide and/or appropriately handling the incubation time.

\section{Conclusion}

In this experiment, we have successfully developed a simple detection method to observe enzymatic reaction of catalase using liquid crystal droplet patterns. A distinctive orientation change of LCs was made after transferring hydrogen peroxide onto the patterns. As aldehydes embedded in LCs were oxidized by hydrogen peroxide, amphiphilic molecules (dodecanoate) were formed. These molecules aligned themselves perpendicularly at the interface, having their hydrophobic tails toward the LCs and their polar heads toward the aqueous phase. As dodecanoate changed their orientation from random to such, LCs started to change their orientation to fit together with dodecanoate. This phenomenon induced LC droplet patterns to be shown as dark cross instead of bright fan-shaped. However, when pre-incubated mixture of catalase and hydrogen peroxide was added, no orientational change happened. This suggests catalase has successfully hydrolyzed hydrogen peroxide to a significant amount and that it stopped aldehydes from aligning at the surface. By this, we could monitor the presence of CAT in the aqueous phase. Detection limit for CAT found in this reaction was 1 fM. By increasing surface area sufficiently, we demonstrated that LC droplet patterns can be highly sensitive detector not only for CAT but also for many other biochemical events. This experiment shows that this system carries a high compatibility as a biosensor.

Acknowledgments. This work was supported by the GRRC program of Gyeonggi province (Grant No. GRRC Gachon 2013-B02).

\section{References}

1. Tao, Z.; Raffel, R. A.; Souid, A. K.; Goodisman, J. Biophysical J. 2009, 96, 2977.

2. Bolwell, G. P.; Woljtasek, P. Physiol. Mol. Plant Pathol. 1997, 51, 347.

3. Apel, K.; Hirt, H. Annu. Rev. Plant Biol. 2004, 55, 79.

4. Miller, E. W.; Dickinson, B.C.; Chang, C. J. Proc. Natl. Acad. Sci. USA 2010, 107, 15681.

5. Xiao, Y.; Ju, H.-X.; Chen, H.-Y. Analytica Chimica Acta 1999, $391,73$.

6. Karyakin, A. A.; Karyakina, E. E. Russ. Chem. Bull. Int. Ed. 2001, $50,1811$.

7. Mattos, I. L.; Shiraishi, K. A.; Braz, A. D.; Fernandes, J. R. Quim. Nova 2003, 26, 373.

8. Huang, K.-J.; Niu, D.-J.; Liu, X.; Wu, Z.-W.; Fan, Y.; Chang, Y.F.; Wu, Y.-Y. Electrochimica Acta 2011, 56, 2957.

9. Aizawa, M.; Karube, I.; Suzuki, S. Analytica Chimica Acta 1974, $69,431$.

10. Murthy, M. R. N.; Reid III, T.; Sicignano, A.; Tanaka, N.; Rossmann, M. G. J. Mol. Biol. 1981, 152, 465.

11. Aebi, H.; Suter, H. Adv. Hum. Genet 1971, 143.

12. Goth, L.; Eaton, J. W. Lancet 2000, 356, 1820.

13. Kobayashi, M.; Sugiyama, H.; Wang, D.-H.; Toda, N.; Maeshima, Y.; Yamasake, Y.; Masuoka, N.; Yamada, M.; Kira, S.; Makino, H. Kidney International 2005, 68, 1018.

14. Ashley, J.; Li, S. F. Y. Biosensors and Bioelectronics 2013, 48, 126.

15. Futo, P.; Markus, G.; Kiss, A.; Adanyi, N. Electroanalysis 2012, 24, 107.

16. Cohen, C. B.; Weber, S. G. Anal. Chem. 1993, 65, 169.

17. Hu, Q.-Z.; Jang, C.-H. Journal of Biotechnology 2012, 157, 223. 
18. Adgate, J. L.; Bartekova, A.; Raynor, P. C.; Griggs, J. G.; Ryan, A. D.; Acharya, B. R.; Volkmann, C. J.; Most, D. D.; Lai, S.; Bonds, M. D. J. Environ. Monit. 2009, 11, 49.

19. Gupta, V. K.; Skaife, J. J.; Dubrovsky, T. B.; Abbott, N. L. Science 1998, 279, 2077.

20. Brake, J. M.; Abbott, N. L. Langmuir 2007, 23, 8497.

21. Liao, S.; Qiao, Y.; Han, W.; Xie, Z.; Wu, Z.; Shen, G.; Yu, R. Anal. Chem. 2012, 84, 45.
22. Hu, Q. Z.; Jang, C. H. Soft Matter 2013, 9, 5779.

23. Bi, X.; Hartono, D.; Yang, K.-L. Adv. Funct. Mater. 2009, 19, 3760.

24. Bi, X.; Yang, K.-L. Biosens Bioelectron. 2010, 26, 107.

25. Hu, Q.-Z.; Jang, C.-H. Talanta 2012, 99, 36.

26. Sato, K.; Hyodo, M.; Takagi, J.; Aoki, M.; Noyori, R. Tetrahedron Letters 2000, 41, 1439.

27. Liu, D.; Jang, C.-H. Sensors and Actuators B 2014, 193, 770. 